\title{
Individualizing goals for users of externally powered partial hand prostheses
}

\author{
Lynsay Whelan, MS, OTR/L; ${ }^{*}$ Sharon Flinn, PhD, OTR/L, CHT; ${ }^{2}$ Nathan Wagner, LPO, CPO, OTR/L ${ }^{3}$ \\ ${ }^{1}$ Occupational Therapy, Touch Bionics, Hilliard, $\mathrm{OH} ;{ }^{2}$ School of Health and Rehabilitation Sciences, The Ohio State \\ University, Columbus, $\mathrm{OH} ;{ }^{3}$ Prosthetics, Touch Bionics, Hilliard, $\mathrm{OH}$
}

\begin{abstract}
Based on recent advances in prosthetic technology available for individuals with partial hand loss or deficiency, research is needed to best ensure the acceptance of these devices. Additionally, this population is subjected to higher risks of overuse syndromes. With improved technological advances in prosthetic devices, clients can engage in more complex activities with less compensation. The purpose of this study was to describe the tasks identified as both difficult and important to individuals with partial hand loss. Recommendations will be provided regarding matching users' individualized goals with considerations in prosthetic design, functional training, programming, and adaptive equipment.
\end{abstract}

Key words: adaptive equipment, functional training, goal setting, limb deficiency, limb loss, multi-articulating prosthetic hand, partial hand deficiency, prosthesis, prosthesis overuse, prosthetic technology.

\section{INTRODUCTION}

Epidemiological data estimate that of the 20,000 new cases of upper-limb loss or deficiency each year, approximately 90 percent occur at the wrist or more distal [1]. Partial hand limb loss or deficiency can have a profound impact on function. The American Medical Association provides an impairment rating of 40 percent hand impairment, 36 percent upper-limb impairment, and 22 percent impairment to the person as a whole when the thumb is amputated at the metacarpophalangeal (MCP) joint [2]. Hand impairment as high as 100 percent can result when there are amputations of the thumb and all fingers at the MCP joints [2]. Since externally powered partial hand prostheses have only been available since 2008, these individuals had previously relied upon the creativity of the prosthetic and rehabilitation team to provide functional solutions. Before 2008, the prosthetic options were passive functional devices, task-specific devices, and some limited mechanical designs. These designs are still available today and can complement an externally powered device as a secondary prosthesis. Also, they can be an appropriate option when the user's needs relate to goals that are contraindicated for an externally powered device, such as exposure to dirt, heavy vibration, or moisture.

Abbreviations: $\mathrm{ADL}=$ activity of daily living; $\mathrm{COPM}=$ Canadian Occupational Performance Measure; DASH = Disability of the Arm, Shoulder and Hand; FPST = Flinn Performance Screening Tool; IADL = instrumental ADL; IRB = Institutional Review Board; $\mathrm{MCP}=$ metacarpophalangeal; $\mathrm{OI}=$ overuse injury; ROM = range of motion; VLA = valued life activities.

*Address all correspondence to Lynsay Whelan, MS, OTR/L; Touch Bionics - Occupational Therapy, 3455 Mill Run Dr, Suite 310, Hilliard, OH 43026; 614-767-9520; fax: 614-7101524. Email: lynsay.whelan@touchbionics.com

http://dx.doi.org/10.1682/JRRD.2013.08.0181 
Passive functional devices include any device designed with static components that have no inherent movement within the device. Silicone restoration prostheses are considered passive functional because they allow the user to support, push, pull, and stabilize objects as well as provide psychological benefits to the user because the device resembles the anatomical details of the missing fingers or hand. Opposition posts are also passive functional devices and allow remnant fingers to oppose against a static armature or platform on the prosthesis. Another option for prosthetic partial hand use is the task-specific device. These devices are designed to help the user participate in a specific activity and can include everything from cutlery and tools, to billiards and fishing. Mechanical fingers have become more advanced in recent years and operate through the harnessing of proximal joints and anatomy. Because mechanical fingers rely on the motion of the user, their use is dependent upon the presentation, strength, and range of motion (ROM) of the wearer. Finally, externally powered partial hand prostheses provide multiarticulating motorized digits that are powered by batteries. These devices provide the highest grasp and pinch forces of the previously mentioned devices and can simulate the pinch and grip patterns of the human hand.

In a research study comparing Vietnam veterans to the more recent veterans from the conflicts in Iraq and Afghanistan, the more recent veterans reported that, of all the levels of upper-limb loss, partial hand limb loss had the greatest impact on their quality of life [3]. In this same study, the partial hand veterans from Iraq and Afghanistan were the least likely to use their prosthesis in the completion of activities of daily living (ADLs) [3]. These results are similar to research results from Australia, where individuals with various levels of upper-limb loss were asked to rate their function using the Disability of the Arm, Shoulder and Hand (DASH). In this study, persons with partial hand limb loss reported higher levels of disability than those with major unilateral upper-limb amputations [4]. Data for both of these studies were collected before the availability of externally powered partial hand prosthetic options.

The lack of personalized prosthetic training can lead to the rejection of hand prostheses and contribute to the development of overuse injuries (OIs). Persons with upper-limb amputations are at risk for developing OIs sometime in their lifetime [5]. As many as 50 percent of persons with unilateral upper-limb amputations reported OIs within 2-5 yr postinjury for diagnoses such as epi- condylitis, tenosynovitis, trigger finger, and carpal tunnel syndrome [6]. Moreover, the unaffected upper limbs have higher than expected OI pain symptoms in the shoulder (45\%), elbow (28\%), wrist (28\%), and hand (23\%) [7].

Compared with individuals with transradial levels of amputation, persons with partial hand limb loss or deficiency are uniquely challenging for prosthetists and therapists. Oftentimes, remaining digits may have musculoskeletal comorbidities such as hyper- or hyposensitive tissue, decreased ROM, adherent scar tissue, decreased circulation, neuromas, or burns. Consideration must be made for maintaining active ROM at remaining joints. Additionally, the team must consider how the remaining fingers will interact with the mechanical digits, how the alignment of the mechanical digits will affect function, and how to optimize control of the device with multiple grasp patterns and coordinated movements. This coordinated movement begins with the ability to pinch and grasp objects and evolves into high-demand ADLs, instrumental ADLs (IADLs), work, and leisure tasks through functional training. In order to provide this previously underserved population with functional solutions, it is important to ensure that the individual's goals are identified and addressed.

Literature on goal setting has identified the importance of ensuring meaningfulness to the client rather than substituting the clinician's values onto the client [8]. As this relates to upper-limb prostheses, the presence of functional training increases the rate of integration of the device into the individual's daily life from 50 to 90 percent [9]. The quality of training and level of experience by the prosthetic and occupational therapy team members can outweigh the impact of being fitted late when one is looking at reducing rejection rates of upper-limb prostheses [10].

Other studies have explored how hand prostheses affect a user's ability to engage in various ADLs. However, prior studies have failed to identify the priority activities of the prosthetic user and to ensure that the design of the prosthesis contributes to the successful performance of those goals. The purpose of this study, therefore, was to identify those tasks that individuals with partial hand limb loss or deficiency found both difficult to perform and important. In practice, this information leads to personalized modifications for individuals with prosthetic hands to ensure accomplishment of priority tasks. These modifications will be described in the "Discussion" section from the prosthetist's and occupational therapist's perspective. 


\section{METHODS}

\section{Participants and Recruitment}

The study was implemented at the Touch Bionics Center of Excellence located in central Ohio. The potential subjects were being fitted for their Touch Bionics i-digits partial hand prostheses as a result of hand amputations, either acquired or congenital, and involving one or more digits of the hand. Subjects were recruited through convenience sampling from all persons referred from June 2010 to November 2011. Eligible clients were invited to participate in the study and consented.

\section{Measures}

\section{Demographic Information}

A survey questionnaire assessed demographic information for sex, age, educational level, marital status, ethnicity, home environment, employment status, level of work demands, amputation level, time since amputation, cause of amputation, dominance pre- and postinjury, and changes in body functions. Categorical responses for each item were collected.

\section{Screen of Activity Limitations}

The Flinn Performance Screening Tool (FPST) was selected as a tool that identifies the valued life activities (VLAs) of individuals recovering from an injury. Defined as a wide range of activities that individuals perform on a daily basis, performance of VLAs has been strongly linked to psychological well-being, satisfaction with function, and quality of life [11]. The client selects important and problematic activities from a comprehensive card sort of 301 photographs representing 25 categories of occupation described in the "Occupational Therapy Practice Framework" [12]. Face validity was established from 200 orthopedic and rheumatology clients. Psychometric properties have been established for test-retest reliability, internal consistency, interrater agreement, and construct validity for persons with orthopedic disorders [13]. Figure 1 provides examples from the FPST.

A dichotomous rating scale was used for the FPST, where a score of 1 indicated that the activity was important but problematic to the subject and a score of 0 indicated that the activity was not important or not problematic. Of the important but problematic items, subjects were asked to rank the top five priorities. The scores on the FPST range from $0-144$ for the ADL subscale, $0-157$ for the IADL subscale, and 0-301 for the total scale.
The ranked activities and the number of important but problematic items on the FPST were calculated on each subject. The higher the number of important and problematic scores on the FPST, the greater the level of self-reported disability. Due to the large number of FPST items, ranking of five priority items was used to identify common concerns by the subjects.

\section{Procedures}

The study protocol was approved by The Ohio State University Institutional Review Board (IRB)-Biomedical Sciences. Graduate students from the Occupational Therapy program were trained in the consent process and administration of the demographic survey and the FPST. Two students completed the data collection to avoid possible bias by the investigative team. The identification number for each problematic FPST item was then entered into an Excel (Microsoft Corporation; Redmond, Washington) spreadsheet for analysis.

\section{Analytic Analysis}

Descriptive analysis was used to examine the demographic data. Frequency distributions were calculated for the total number and five priority FPST items.

\section{RESULTS}

\section{Sample Characteristics}

Fifteen participants with partial hand amputations completed the study. The demographic characteristics are reported in Table 1.

\section{Activity Limitations}

The participants reported limitations in all categories of the FPST. Based on the total number of 301 FPST items, an average of 18.51 items was recorded per subject (standard deviation $=18.51$, range $=0-62$ ). Ten activity limitations were identified by 60 percent or more of the cohort as important but difficult to perform. Table 2 reports the most commonly reported activity limitations.

\section{DISCUSSION}

The goal of this descriptive study was to identify important but problematic tasks commonly reported by individuals with partial hand limb loss or deficiency. 


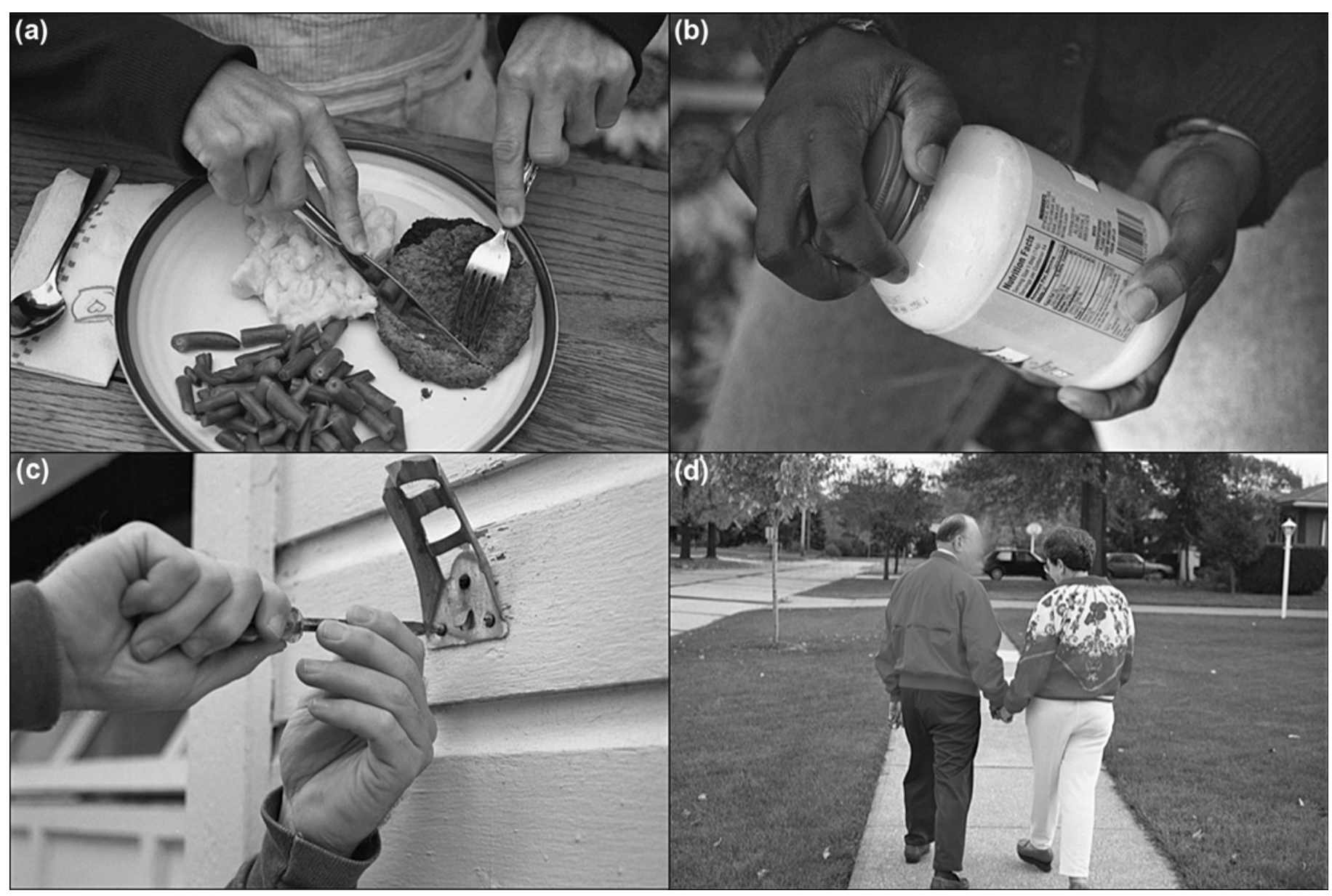

Figure 1.

Example Flinn Performance Screening Tool items: (a) cut meat, (b) open/close jar, (c) perform minor repairs, and (d) hold hands.

Systematic administration of one client-centered measure, the FPST, was done before prosthetic fitting to target priority tasks. Sixty percent or more of the cohort reported 10 common activities that were important and difficult to perform. In addition, a personalized goal profile for each participant was developed that included as many as 62 tasks. Utilizing this kind of data fosters a client-centered approach to prosthesis delivery for persons with partial hand limb loss or deficiency. This is a new and in-depth way to consider a user's functional goals before prosthetic fitting, which ensures that the design and operation of the prosthesis meet the unique needs of the user. Identifying what is meaningful to a client can influence the fitting process in four ways: (1) design of the prosthesis, (2) programming of the prosthesis, (3) training of prosthesis use, and (4) recommendation of adaptive equipment.
Prosthetic design can be influenced by knowing the unique functional demands of the end user. For example, cutting meat was the primary goal for 93 percent of the participants. Each prosthesis was tested and modified as needed to complete this task. Modifications included using a notch or proximal anchor point for the knife or fork, changing prosthetic digit alignment, and adding material linings with a high coefficient of friction, as seen in Figure 2.

Programming the software of the i-limb digits prosthesis (Touch Bionics; Hilliard, Ohio) was also influenced by the data collected. The software has the ability to change grip patterns, the grip and pinch force generated through vari-grip pulsing, and the sensitivity of inputs. Changing grip patterns was helpful in accommodating goals concerning tool use, improving line of site, and customizing grips based on needs of the task, as seen in Figure 3. For 
Table 1.

Demographic information for persons with partial hand prosthesis $(n=15)$.

\begin{tabular}{|c|c|}
\hline & Finding \\
\hline Male, $n(\%)$ & $11(73.3)$ \\
\hline Age, Mean \pm SD (range) & $37 \pm 11.88(19-55)$ \\
\hline \multicolumn{2}{|l|}{ Ethnicity, \% } \\
\hline \multicolumn{2}{|l|}{ Caucasian } \\
\hline \multicolumn{2}{|l|}{ African American } \\
\hline \multicolumn{2}{|l|}{ Education, \% } \\
\hline \multicolumn{2}{|c|}{$\begin{array}{l}\text { High School Graduate/General Educational } \\
\text { Development }\end{array}$} \\
\hline \multicolumn{2}{|c|}{ Advanced Training/Certification } \\
\hline \multicolumn{2}{|c|}{ Some College, No Degree } \\
\hline \multicolumn{2}{|l|}{ Associates Degree } \\
\hline \multicolumn{2}{|l|}{ Marital Status, \% } \\
\hline \multicolumn{2}{|l|}{ Single } \\
\hline \multicolumn{2}{|l|}{ Married } \\
\hline \multicolumn{2}{|l|}{ Divorced } \\
\hline \multicolumn{2}{|l|}{ Hand Dominance, \% } \\
\hline \multicolumn{2}{|l|}{ Right Always } \\
\hline \multicolumn{2}{|l|}{ Left Always } \\
\hline \multicolumn{2}{|l|}{ Switched Dominance } \\
\hline \multicolumn{2}{|l|}{ Living Arrangement, \% } \\
\hline \multicolumn{2}{|c|}{ Lives Alone, Needs No Assistance } \\
\hline \multicolumn{2}{|c|}{ Lives Alone, Has Outside Assistance } \\
\hline \multicolumn{2}{|l|}{ Lives With Others } \\
\hline \multicolumn{2}{|l|}{ Occupation, \% } \\
\hline \multicolumn{2}{|l|}{ Student } \\
\hline \multicolumn{2}{|l|}{ Homemaker } \\
\hline \multicolumn{2}{|l|}{ Paid Employee } \\
\hline \multicolumn{2}{|l|}{ Unemployed } \\
\hline \multicolumn{2}{|l|}{ Physical Work Demands, \% } \\
\hline \multicolumn{2}{|c|}{ Sedentary (<10 lb occasionally) } \\
\hline \multicolumn{2}{|c|}{ Light (20 lb maximum, 10 lb frequently) } \\
\hline Medium (50 lb maximu & \\
\hline Heavy (100 lb maximuı & \\
\hline $\begin{array}{l}\text { Very Heavy (>100 lb m } \\
\text { frequently) }\end{array}$ & \\
\hline Date of Injury, \% & \\
\hline$<12$ mo & \\
\hline$\geq 13$ mo & \\
\hline Cause of Amputation, \% & \\
\hline Birth & \\
\hline Work Related & \\
\hline Non-Work Related & 7.1 \\
\hline
\end{tabular}

Table 2.

Common activity limitations from Flinn Performance Screening Tool $(n=15)$

\begin{tabular}{lc}
\hline & \% Limitation \\
\hline Cut Meat & 93 \\
Peel Vegetables & 80 \\
Trim Nails & 73 \\
Fasten Buttons & 67 \\
Serve Food & 60 \\
Tie Rubbish & 60 \\
Care for Toenails & 60 \\
Open Packages & 60 \\
Wash Glasses & 60 \\
Carry Bulky Items & 60 \\
\hline \hline
\end{tabular}

example, increased pinch/grip force was useful in tying shoelaces, tying garbage bags, opening packages, and opening jars and in other tasks requiring extra force to prevent the held object from slipping, such as those in Figure 4. Adjusting the sensitivity of the inputs was also crucial in maintaining proportional control when holding delicate objects and for ensuring no inadvertent signals were processed while carrying objects.

Individualized training sessions for functional training with the prosthesis were highly influenced by the priorities of the user. When difficult but important bilateral tasks were identified, it was necessary to educate the patient on using the prosthesis as a "helper hand" rather than the dominant hand when the individual was involved unilaterally. In this way, the prosthesis became the component of the bilateral task that stabilized, held, and positioned objects, such as in Figure 5. Proper positioning of thumb rotation was significantly influenced by the goals selected by the user. Given that the thumb is manually rotatable, it was important the patient prepositioned the thumb in the right amount of opposition before attempting to complete the identified tasks. Training sessions were enhanced by repetitive practice of specific, meaningful tasks to foster habitual and correct use of the prosthesis. In addition, participants were more likely to engage in therapy and to see the benefits of using their prostheses when applied to their specific needs.

Only when prosthetic design, programming, and training efforts failed to overcome the users' activity limitations was specific adaptive equipment recommended. For example, trimming finger and toenails was a common activity limitation expressed by the participants that responded best 
to adaptive equipment. One method of improving independence in nail care was the application of low-temperature plastic to the end of clippers shown in Figure 6. This modification improved the mechanical advantage of the task and allowed the remaining fingers or prosthetic digits better control of the tool. Another commonly used piece of adap-

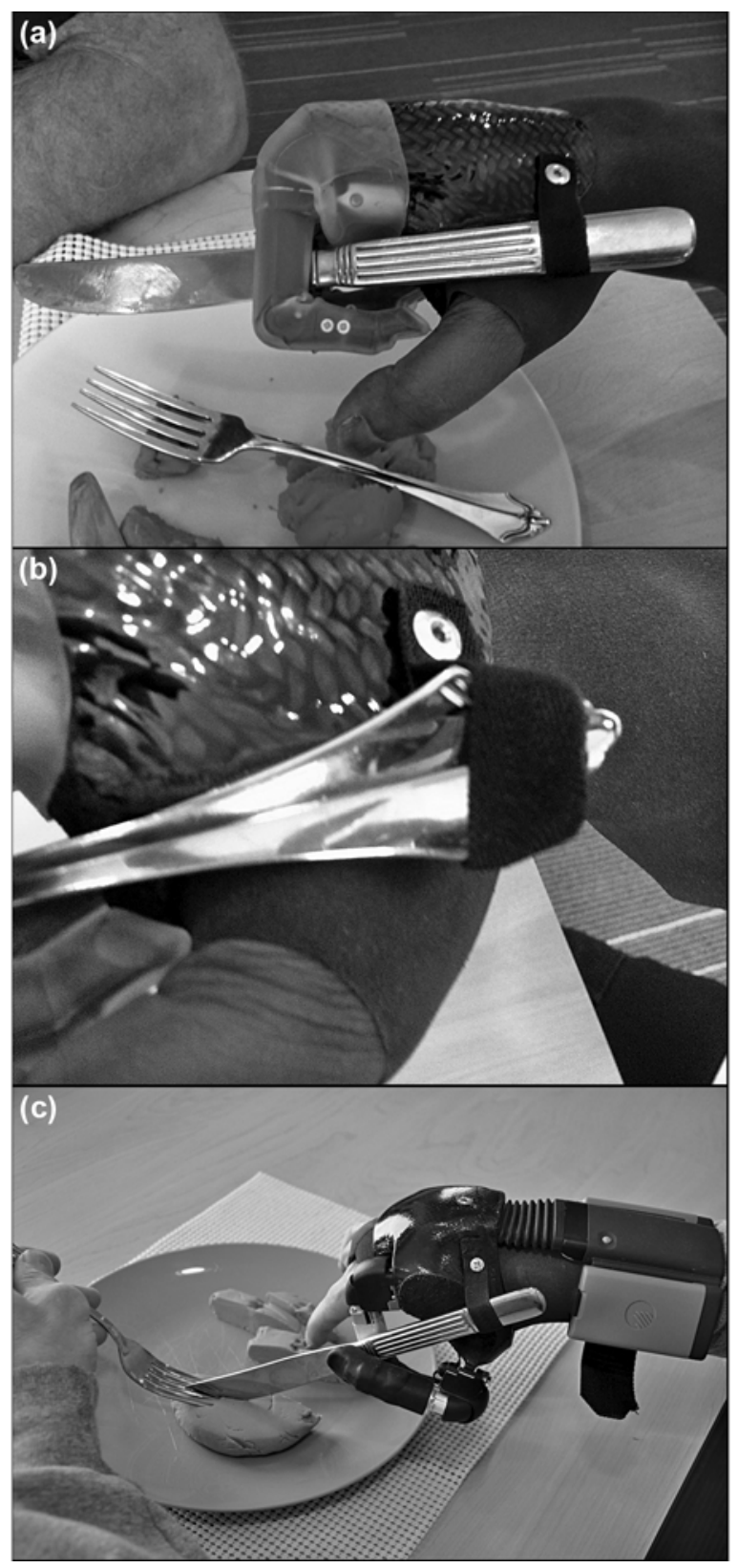

Figure 2.

Examples of modifications for holding utensils: (a) addition of Dacron strap to stabilize knife, (b) strap holding fork (close up), and (c) addition of leather to better stabilize. tive equipment was Dycem (Warwick, Rhode Island) to decrease the sliding of objects. Dycem was commonly used under a plate to prevent the plate from sliding while the user was cutting meat.

In addition to improving the fitting process, the use of client-centered data can represent more accurate disability levels and realistic gains in functional outcomes. Since there are limited numbers of studies on prosthetic use, it was important to compare the functional limitations identified by two standardized outcome measures in previous studies with the client-centered list described by prosthetic users in this study. The activities measured in these outcomes measures did not correspond well with

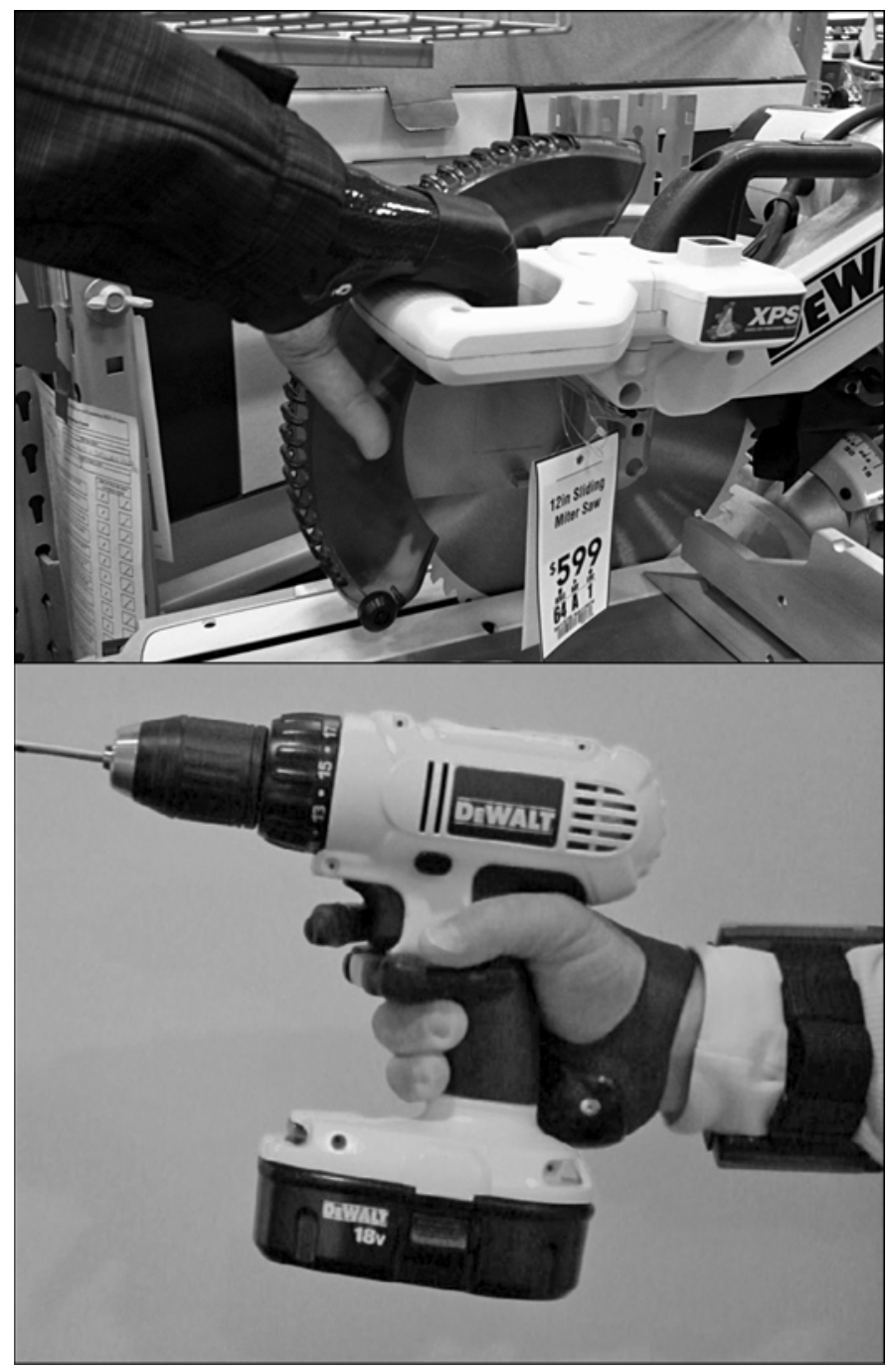

Figure 3.

Examples of improved tool use based on grip patterns available. 


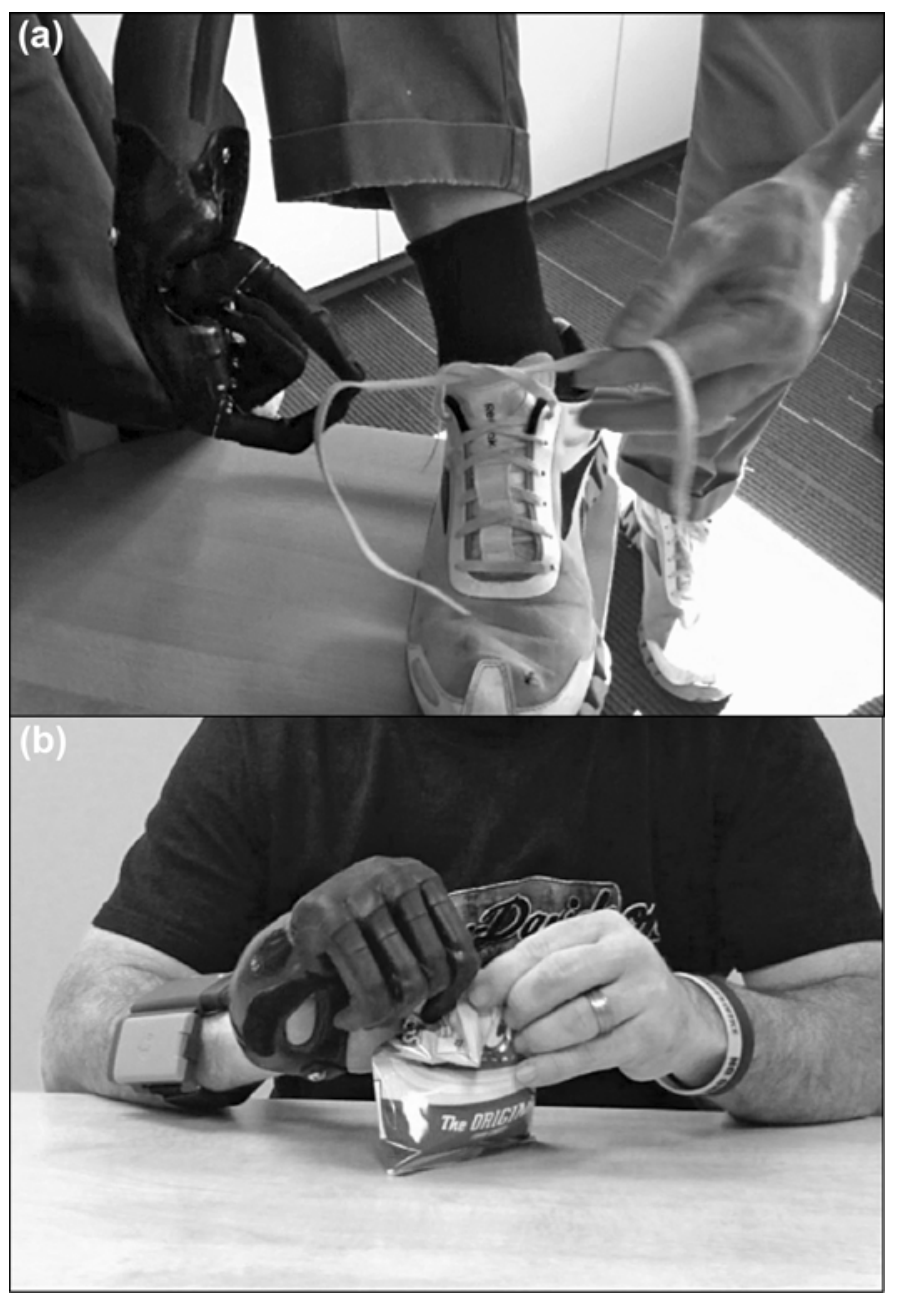

Figure 4.

Examples of tasks requiring additional grip force to complete: (a) tying shoelaces and (b) opening packages.

those identified in our study. Of the 21 activities listed on the 2010 version of the DASH [4], only 1 item (cutting meat) was reported as important and problematic by our participants. Similarly, of the 21 activities listed on the Upper-Limb Activity Measure [3], only 2 items were reported (cutting meat and peeling vegetables). These findings suggest that many VLAs are not reflected in standard outcome measures, especially for persons with partial hand amputations. Unfortunately, practitioners may underestimate the upper-limb disability caused by partial hand limb loss or deficiency if more in-depth analysis of the tasks that are important to users are not identified in the assessment.

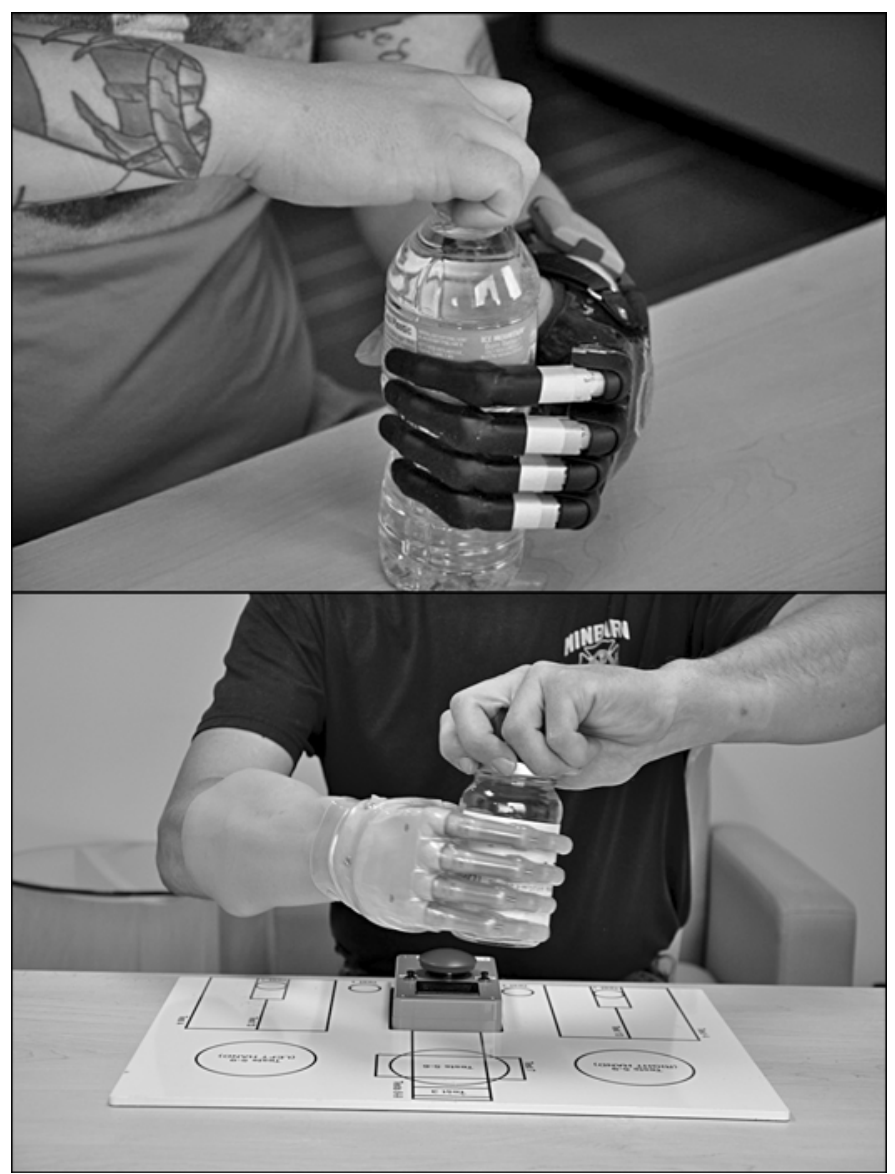

Figure 5.

Examples of bilateral tasks using prosthesis as helper hand.

Future studies are needed to understand the effect of personalized intervention programs on the quality of prosthetic devices and potential functional outcomes. Of particular interest for persons with partial hand amputations would be comparison studies between standard and personalized interventions on satisfaction levels, rates for prosthetic rejection, compensatory differences, overuse problems, and cost efficiency. Similar studies utilizing targeted activities from the client can benefit from applying standardized assessment rating scales, such as the Canadian Occupational Performance Measure (COPM) [14], to obtain change scores for importance and satisfaction of activity performance. The COPM is a semistructured interview and relies on individuals recalling relevant tasks that they find difficult. The pictures in the FPST cue them to remember activities, like raking the yard, that they may not have done in several years. While the COPM was not used in this study, ranked items from the FPST complement the 


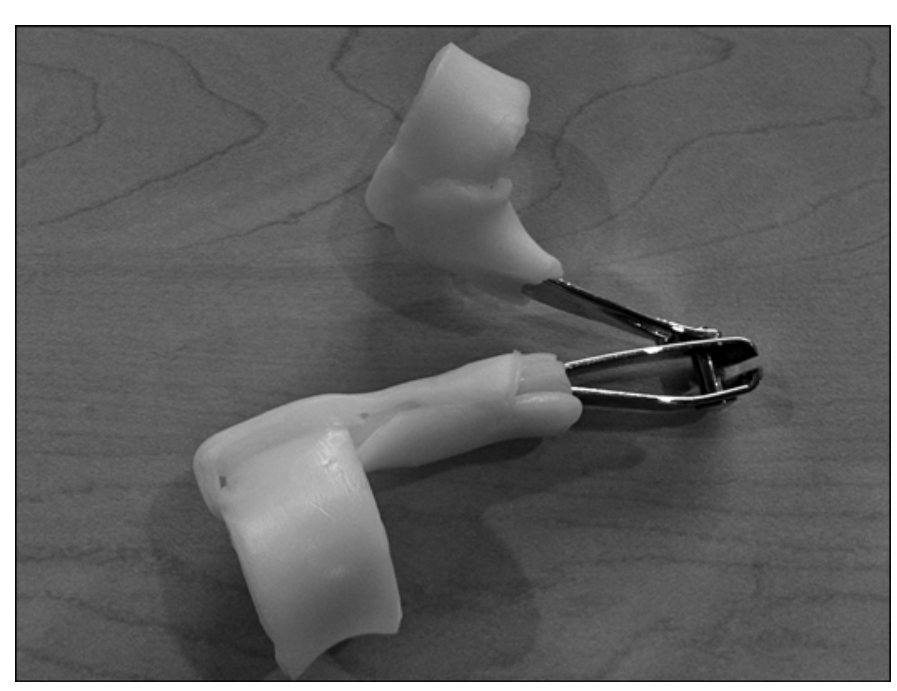

Figure 6.

Example of adaptations made to tools to improve use with prosthesis.

COPM format and rating scales. Gain scores can be calculated to track the users' progress in mastering the use of their prosthesis.

\section{LIMITATIONS}

Because the participants of the study represent a convenience sample, threats to internal validity are possible and results may not be indicative of the entire population under study. A small sample size $(n=15)$ collected from only one facility, regardless of the fact the subjects come from various parts of the country, might potentially confound the data collected in the study.

The small sample size of 15 participants represents young, Caucasian males who are educated and employed. The majority of the participants had unilateral limb loss or deficiency, were seen more than 13 mo postinjury, and most frequently lived with others. These demographic characteristics may influence the type and number of activity limitations reported by the population of persons with partial hand amputation.

The FPST was administered by trained occupational therapy students and has adequate psychometric properties. However, the use of self-reported data as the only measure of functional limitations might pose additional threats to internal validity.

\section{CONCLUSIONS}

Individuals with partial hand limb loss or deficiency report an average of 18 important and problematic tasks, with a range of $0-62$. While some tasks were consistently reported by a majority of clients, there was also large variability among other priority tasks. This study suggests the need to identify and personalize the care of individuals with partial hand amputations for improved prosthetic fabrication and functional outcomes. The ability to address priority tasks in the design and programming of and training with a partial hand prosthesis shows promise when personalized goals are obtained.

\section{ACKNOWLEDGMENTS}

\section{Author Contributions:}

Literature review: S. Flinn, L. Whelan.

Study concept and design: S. Flinn, L. Whelan, N. Wagner. Acquisition of data: S. Flinn.

Analysis and interpretation of data: S. Flinn.

Drafting of manuscript: L. Whelan, S. Flinn, N. Wagner.

Administrative, technical, or material support: L. Whelan.

Study supervision: L. Whelan, S. Flinn.

Financial Disclosures: The FPST used in the study was developed and copyrighted by Dr. Sharon Flinn, who is affiliated with an organization with a financial interest in the instrument. Nevertheless, the IRB reviewed the research protocol and determined that the use of the FPST did not pose a threat to the participants. The coauthors of this article have no financial interest in the FPST.

Funding/Support: This material was unfunded at the time of manuscript preparation.

Institutional Review: The protocol for the study was approved by The Ohio State University IRB and was conducted in a manner that conformed to the approved protocol.

Participant Follow-Up: The authors do not plan to inform participants of the publication of this study because data was de-identified and participants previously approved for publication of information in aggregate form.

\section{REFERENCES}

1. Dillingham TR, Pezzin LE, MacKenzie EJ. Limb amputation and limb deficiency: Epidemiology and recent trends in the United States. South Med J. 2002;95(8):875-83. [PMID:12190225]

2. Rondinelli RD. Guides to the evaluation of permanent impairment. 6th ed. Chicago (IL): American Medical Association; 2007. p. 441-45.

3. McFarland LV, Hubbard Winkler SL, Heinemann AW, Jones M, Esquenazi A. Unilateral upper-limb loss: Satisfaction and 
prosthetic-device use in veterans and servicemembers from Vietnam and OIF/OEF conflicts. J Rehabil Res Dev. 2010; 47(4):299-316. [PMID:20803400] http://dx.doi.org/10.1682/JRRD.2009.03.0027

4. Davidson J. A comparison of upper limb amputees and patients with upper limb injuries using the Disability of the Arm, Shoulder and Hand (DASH). Disabil Rehabil. 2004; 26(14-15):917-23. [PMID:15497922] http://dx.doi.org/10.1080/09638280410001708940

5. Gambrell C. Overuse syndrome and the unilateral upper limb amputee: Consequences and prevention. J Prosthet Orthot. 2008;20:126-32. http://dx.doi.org/10.1097/JPO.0b013e31817ecb16

6. Jones LE, Davidson JH. Save that arm: A study of problems in the remaining arm of unilateral upper limb amputees. Prosthet Orthot Int. 1999;23(1):55-58. [PMID:10355644]

7. Datta D, Selvarajah K, Davey N. Functional outcome of patients with proximal upper limb deficiency-acquired and congenital. Clin Rehabil. 2004;18(2):172-77.

[PMID:15053126] http://dx.doi.org/10.1191/0269215504cr716oa

8. Eschenfelder V. Shaping the goal setting process in OT: The role of meaningful occupation. Phys Occup Ther Geriatr. 2005;23(4):67-81. http://dx.doi.org/10.1080/J148v23n04 05

9. Durance JP, O'Shea BJ. Upper limb amputees: A clinic profile. Int Disabil Stud. 1988;10(2):68-72.

[PMID:3403501] http://dx.doi.org/10.3109/09638288809164107

10. Resnik L, Meucci MR, Lieberman-Klinger S, Fantini C, Kelty DL, Disla R, Sasson N. Advanced upper limb prosthetic devices: Implications for upper limb prosthetic rehabilitation. Arch Phys Med Rehabil. 2012;93(4):710-17.

\section{[PMID:22464092]}

http://dx.doi.org/10.1016/j.apmr.2011.11.010

11. Katz PP, Morris A, Yelin EH. Prevalence and predictors of disability in valued life activities among individuals with rheumatoid arthritis. Ann Rheum Dis. 2006;65(6):763-69. [PMID:16249225] http://dx.doi.org/10.1136/ard.2005.044677

12. American Occupational Therapy Association. Occupational therapy practice framework: Domain and process 2nd edition. Am J Occup Ther. 2008;62:625-83.

13. Flinn SR, Pease WS, Freimer ML. Score reliability and construct validity of the Flinn Performance Screening Tool for adults with symptoms of carpal tunnel syndrome. Am J Occup Ther. 2012;66(3):330-37. [PMID:22549598] http://dx.doi.org/10.5014/ajot.2012.000935

14. Law M, Baptiste S, McColl MA, Opzoomer A, Polatajko $\mathrm{H}$, Pollock N. The Canadian Occupational Performance Measure: An outcome measure for occupational therapy. Can J Occup Ther. 1990;57(2):82-87. [PMID:10104738] http://dx.doi.org/10.1177/000841749005700207

Submitted for publication August 14, 2013. Accepted in revised form February 12, 2014.

This article and any supplementary material should be cited as follows:

Whelan L, Flinn S, Wagner N. Individualizing goals for users of externally powered partial hand prostheses. J Rehabil Res Dev. 2014;51(6):885-94. http://dx.doi.org/10.1682/JRRD.2013.08.0181

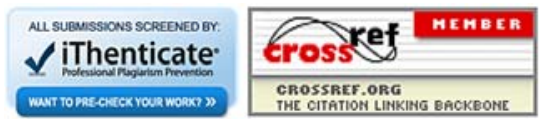


